\title{
Tratamiento endovascular de pseudoaneurisma de la arteria tibial posterior
}

\author{
Endovascular treatment of posterior tibial artery pseudoaneurysm
}

Franco Giannasi Guallart ${ }^{1}$, Ciro D’Aessandro², Laura De Candido ${ }^{3}$, Gustavo Tamashiro ${ }^{4}$, Horacio Maffeo²

\section{RESUMEN}

Los pseudoaneurimas en miembros inferiores son una entidad rara, más aún aquellos localizados a nivel infrapoplíteo; si bien su historia natural es desconocida, los registros describen una gran morbilidad. El constante desarrollo en técnicas de imágenes no invasivas y de mayor sensibilidad ha hecho que su diagnóstico sea cada vez más frecuente. De todos modos, dada su baja incidencia aún no existe un consenso en cuanto a su tratamiento. El objetivo del trabajo es presentar el caso clínico de un varón de 26 años, deportista, con diagnóstico de pseudoaneurisma de arteria tibial posterior derecha de gran tamaño, sintomático por dolor. Dadas las características anatómicas se decidió tratamiento endovascular mediante embolización con técnica "en sándwich" con coils de liberación controlada, con excelente resultado inmediato y en el seguimiento, y sin complicaciones.

Palabras claves: embolización, pseudoaneurisma, coils, tibial posterior.

\section{ABSTRACT}

The pseudoaneurysms in the lower limbs are a rare entity, even the infrapoplitea ones, with unknown natural history, but with great morbidity, their diagnosis became more frequent thanks to increasingly sensitive methods. Given its low incidence and its wide therapeutic range, there is still no consensus regarding its treatment. The aim of the study is to present the clinical case of a 26-year-old male, a sportsman diagnosed with a symptomatic psudoaneurysm of a large posterior tibial artery. Given the anatomical characteristics, endovascular treatment was decided by sandwich embolization with removable coils with excellent results and without complications.

Key words: embolisation, pseudoaneurysm, posterior tibial, coils.

Revista Argentina de Cardioangiología Intervencionista 2019;10(4):0187-0189. https://doi.org/10.30567/RAC1/201904/0187-0189

\section{INTRODUCCIÓN}

El pseudoaneurisma arterial en la extremidad inferior es una entidad poco frecuente, en particular en el segmento infrapoplíteo. Si bien se desconoce su historia natural, sus complicaciones pueden acarrear gran morbimortalidad. Las indicaciones terapéuticas siguen siendo controvertidas en las publicaciones internacionales, aunque en los últimos años las técnicas endovasculares parecen ser una opción.

\section{CASO CLÍNICO}

Presentamos el caso de un paciente de sexo masculino de 26 años, sin factores de riesgo cardiovasculares, ni antecedentes personales. Deportista activo.

Consulta por consultorios externos por presencia de masa pulsátil en región interna de la pierna derecha (Figura 1), asociada a dolor al apoyo que impide continuar con su actividad deportiva e incluso diaria, de más de 1 año de evolución. El paciente niega traumatismo reciente en dicha región. Al examen físico, se constata la presencia de una masa pulsátil, no eritematosa, dolorosa a la palpación, sin presencia de soplo o frémito; se constatan pulsos periféricos positivos con buena perfusión distal. Dadas las sospechas clínicas de pseudoaneurisma, y con el objetivo de descartar otros diagnósticos diferenciales, se

1. Sanatorio Ramón Cereijo

2. Hospital Militar Central, Sanatorio Bernal, Sanatorio Nuevo Berazategui, Sanatorio Pelliza, Sanatorio Ramón Cereijo

3. Hospital Naval Pedro Mallo, CEMIC, Sanatorio Ramón Cereijo

4. Sanatorio Providencia, Clínica Ciudad, Sanatorio Ramón Cereijo

$\triangle$ Correspondencia: Franco Giannasi Guallart, Av Libertador 5505 12B, fmagiannasi@gmail.com

Los autores no declaran conflictos de intereses

Recibido: 16/08/2019| Aceptado: 13/12/2019 realiza angiorresonancia magnética nuclear que evidencia pseudoaneurisma a nivel proximal de la arteria tibial posterior derecha (ATP) de $76 \mathrm{~mm}$ de diámetro transverso x 57 $\mathrm{mm}$ de diámetro longitudinal, resto de las arterias infrapoplíteas permeables, confirmándose el diagnóstico por angiografía digital de miembros inferiores (Figura 2).

Se realiza evaluación conjunta del paciente con el servicio de Cirugía Vascular, decidiéndose, dada la posibilidad anatómica, realizar tratamiento endovascular.

\section{Procedimiento}

Se realiza anestesia local con Xylocaína al 2\% a nivel inguinal y del tobillo derecho. Posteriormente se punza en forma anterógrada la arteria femoral común derecha, y en forma retrógrada y guiada por ecografía la ATP derecha; en ambos accesos se colocan introductores valvulados $5 \mathrm{Fr}$. Se administran 5000 UI de heparina sódica.

Se progresan por ambos accesos catéteres Cobra bajo guías hidrofílicas (Terumo - Japón) de 0,035” y se colocan a nivel proximal y distal de la ATP, a nivel del origen del pseudoa-

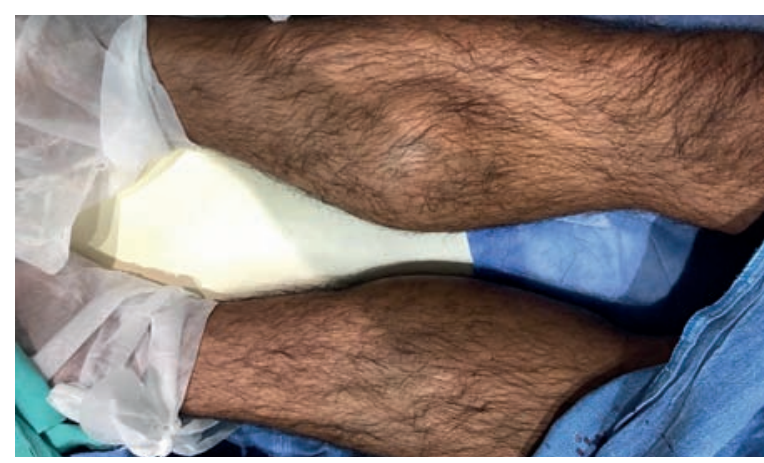

Figura 1. Presencia de masa pulsátil en región interna de pierna derecha, previa al tratamiento endovascular. 


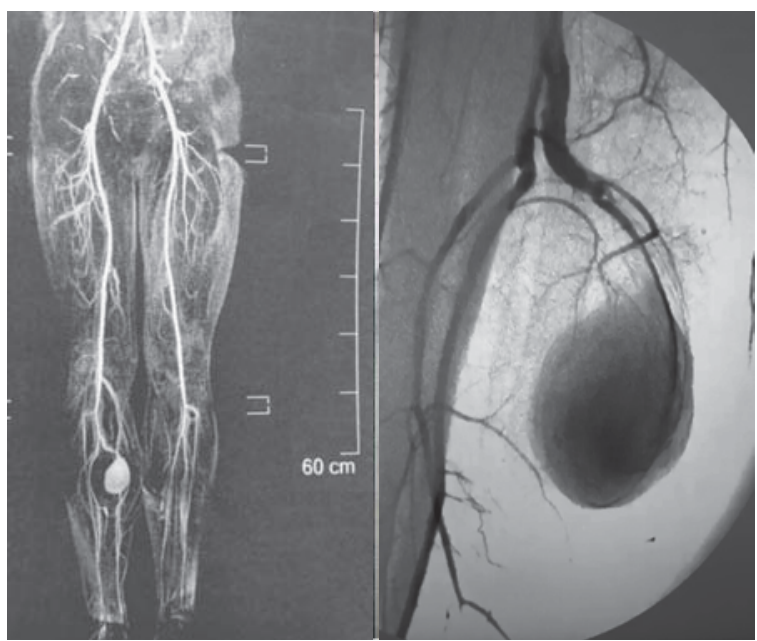

Figura 2. A: Angiorresonancia de miembros inferiores con contraste que evidencia pseudoaneurima a nivel proximal de la arteria tibial posterior (ATP) de $76 \mathrm{~mm}$ de diámetro transverso $\times 57 \mathrm{~mm}$ de diámetro longitudinal, resto de las arterias permeables, sin complicaciones. B: Angiografia de miembro inferior derecho que evidencia pseudoaneurima a nivel proximal de la arteria tibial posterior con flujo en su interior.

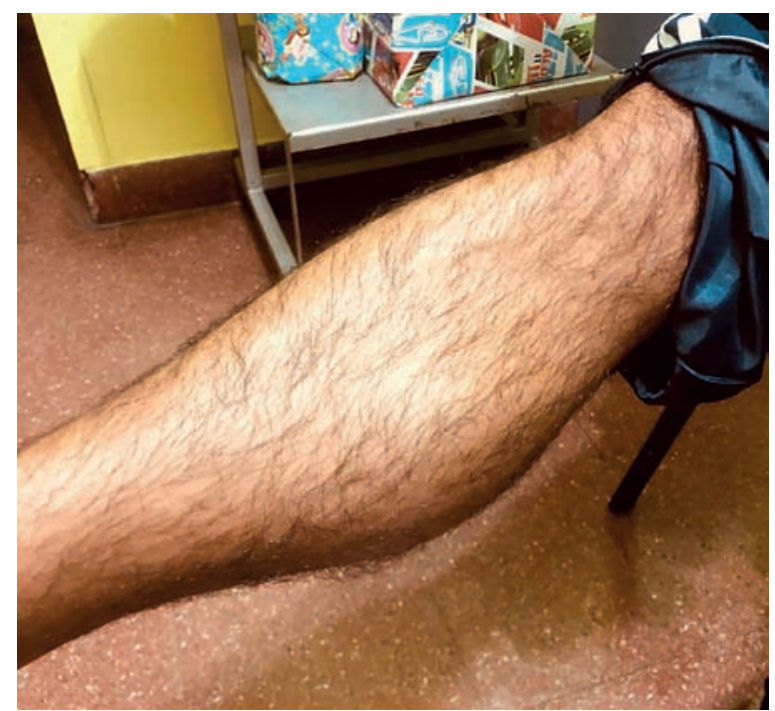

Figura 4. Pierna derecha 30 días postratamiento, sin evidencia de masa.

neurisma. Posteriormente se avanza un microcatéter Rebar 18 (Covidien/Medtronic - USA) ubicado en dicha arteria, proximal y distal al pseudoaneurisma, y se comienzan a liberar los coils Helix ev3 concerto (Covidien/Medtronic - USA) de $8 \mathrm{~mm} \times 30 \mathrm{~cm}$ y de $6 \mathrm{~mm} \times 20 \mathrm{~cm}$, ocluyendo la arteria tibial posterior a nivel proximal; y 2 coils Helix ev3 concerto (Covidien/Medtronic - USA) de $4 \mathrm{~mm}$ x $10 \mathrm{~cm}$ distal al pseudoaneurisma, ocluyendo la arteria eferente (Figura 3).

En el control final se visualiza oclusión total de la ATP derecha y psedudoaneurima sin flujo en su interior (Figura 3). El paciente permanece en control por 24 horas y se le otorga egreso hospitalario con nueva consulta en 30 días, donde se evidencia una clara mejoría de la sintomatología, con franca disminución del tamaño de la masa, y sin pulsatilidad (Figura 4).

\section{DISCUSIÓN}

La incidencia de pseudoaneurisma localizado en la arteria tibial posterior es muy baja, solo documentada en los reportes de casos, por lo cual no existe un verdadero consenso para su tratamiento'.

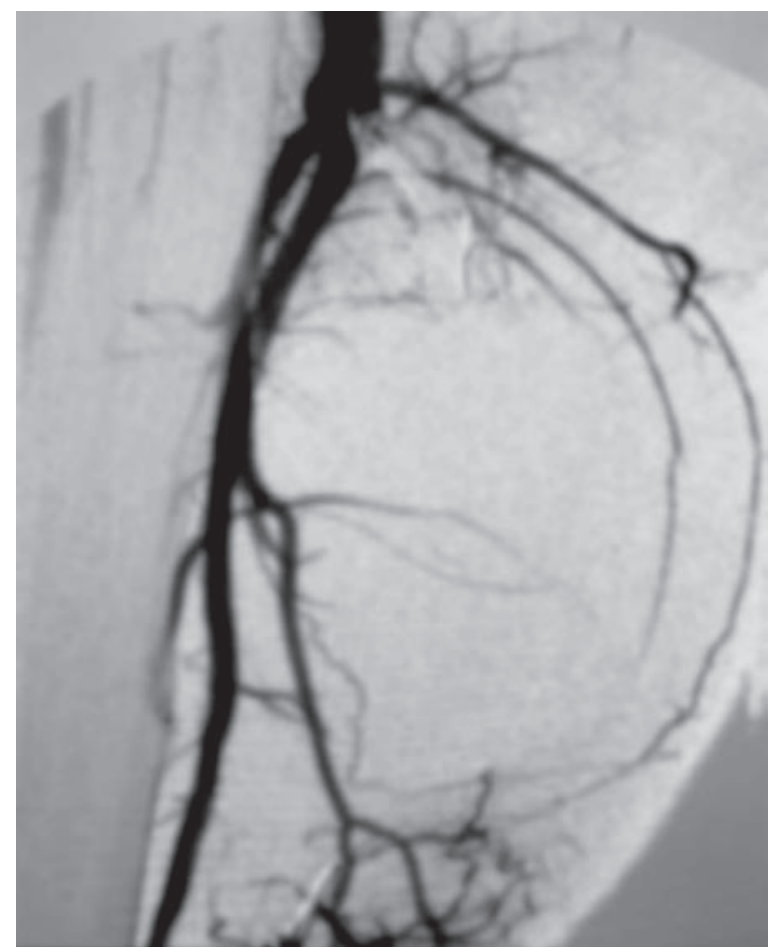

Figura 3. Angiografía posembolización con coils, donde se observa exclusión del pseudoaneurisma.

Las principales causas que conducen a la formación de aneurismas de las extremidades son congénita, traumática, degenerativa, micótica, colagenopatía y dilatación postrombectomia ${ }^{7}$ La forma de presentación más frecuente es la masa pulsátil, siendo su tamaño limitado por la elasticidad de los tejidos circundantes; los síntomas difieren en cada caso, llegando incluso a ser asintomáticos ${ }^{2}$.

Hay poco conocimiento de su historia natural. La trombosis o embolia y la ruptura han sido reconocidas como complicaciones que amenazan las extremidades ${ }^{4}$.

El advenimiento de nuevas técnicas de imágenes con mayor sensibilidad para la enfermedad asintomática ha permitido un diagnóstico más frecuente de pseudoaneurismas. La angiografía convencional es el estándar de referencia para el diagnóstico ${ }^{3}$. La mayoría de las publicaciones recomiendan observar pseudoaneurismas pequeños, asintomáticos, y tratarlos solo si aumentan de tamaño o se vuelven sintomáticos. Sin embargo, el riesgo de rotura espontánea de pseudoaneurismas es muy alta, independientemente de su tamaño. Por lo tanto, algunos autores creen que el tratamiento definitivo debe ser administrado en todos los casos ${ }^{3-4}$.

En la actualidad el tratamiento de los pseudoaneurimas abarca desde una terapéutica conservadora o no invasiva como la compresión o la infusión de trombina guiadas por ecografía ${ }^{5}$ hasta un tratamiento quirúrgico el cual incluye la resección del pseudoaneurisma, con anastomosis termino-terminal o, inclusive, la amputación del miembro ${ }^{6}$.

En los últimos años hubo un gran crecimiento con respecto al manejo endovascular siendoel principal objetivola exclusión del pseudoaneurisma de la circulación sistémica. Esto se puede lograr ralentizando el flujo dentro del pseudoaneurisma (stent forrado o coils); induciendo trombosis (coils y líquidos embólicos); con uso de tapón vascular (plug) y estimulando la inflamación (coils y agentes líquidos) ${ }^{8}$.

Los principales puntos para considerar por el intervencionista antes de elegir la técnica son: el tamaño del pseudoaneuri- 
ma, las características de su cuello, la arteria aferente, sus colaterales, y su localización ${ }^{8}$.

Dadas las características anatómicas en nuestro paciente, el cual presentaba un cuello corto y estrecho, con presencia de arteria aferente y eferente, la técnica elegida fue de embolización "en sándwich" con coils de liberación controlada; la misma consiste en la oclusión de manera proximal y distal al pseudoaneurisma, embolizando la arteria aferente y eferente, con una tasa de éxito reportada mayor al $90 \%{ }^{8}$.

Otras técnicas descriptas para la embolización con coils en un pseudoaneurisma incluyen la oclusión del mismo mediante el relleno del saco y la embolización proximal ${ }^{3}$. Rellenar el saco mediante el uso de colis se logra en el $80-90 \%$ de las veces y mantiene permeable la arteria madre; su principal riesgo es la ruptura secundaria del pseudoaneurima. La embolización proximal es de preferencia en arterias terminales como en el caso de las arterias renales, generando una oclusión incompleta en aquellos pseudoaneurismas con colaterales ${ }^{9}$. El resto de las técnicas descriptas fueron descartadas por las características del pseudoaneurisma y anatómicas del paciente.

\section{BIBLIOGRAFÍA}

1. Canbaz S, Sunar H, Yuksel V, Duran E. Pseudoaneurysm of the posterior tibial artery as a complication of thrombectomy. J Cardiovascular Surg 2004:45:87-8.

2. Bose D, Hauptfleisch J, McNally M. Delayed pseudoaneurysm caused by distal locking screw of a femoral intramedullary nail. A case report. J Orthop Trauma 2006;20(8):584-6.

3. Saad NE, Saad WE, Davies MG, et al. Pseudoaneurysms and the role of minimally invasive techniques in their management. Radiographics 2005;25 Supp/ 1:S173-89.

4. Kato T, Takagi H, Sekino S, et al. Dorsalis pedís artery true aneurysm due to atherosclerosis: Case report and literature review. J Vasc Surg 2004:40:1044-8.
5. La Perna L, Olin JW, Goines D, Childs MB, Ouriel K. Ultrasound-guided thrombin injection for the treatment of post catheterization pseudoaneurysm.

6. Agha RA, Muneer H, Habib M. Posterior Tibial Artery Aneurysm. Bahrain Med Bull 2016;38(2): 13-5.

7. Flores Izquierdo G, Gutiérrez Vogel S. Limb aneurysm. J Cardiovasc Surg 1973:278-84

8. Arata MA, Cope C. Principles used in the management of visceral aneurys ms. Tech Vasc Intervent Radiol 2000;3:124-9.

9. Ikeda O, Nakasone Y, Tamura Y, Yamashita Y. Endovascular management of visceral artery pseudoaneurysms: transcatheter coil embolization using the isolation technique. Cardiovasc Intervent Radiol 2010;33:1128-34. 